\title{
More knowledge of worldwide incidence and epidemiology of spinal cord injury: data from the United States military
}

\author{
JJ Wyndaele, Editor-in-Chief \\ Antwerp University Hospital, Antwerp, Belgium \\ E-mail: spinalcord@uza.be
}

Dear Spinal Cord reader,

All those who participated in the ISCoS annual meeting organized together with ASIA in Washington DC will agree that it was a very good event with nice scientific sessions, a good atmosphere and ample discussions. For those who could not be present, the reports of the ISCoS committees will shortly be available on the ISCoS website, while scientific work presented at the occasion will continue to appear in Spinal Cord in the following months.

The review by Khan et al. showed that subjects with chronic spinal cord injury (SCI ) develop abnormal platelet function, resulting in the production of atherogenic and thrombogenic factors because the $\mathrm{PGI}_{2}$ and insulin receptors on their platelets are impaired, thrombin generation and platelet derived growth factor release are elevated, insulin-induced nitric oxide production by platelets is markedly impaired, and a circulating antibody blocks the antithrombotic effect of both insulin and $\mathrm{PGI}_{2}$ receptors. This antibody may be one of the pathologic mediators of the increased incidence of coronary heart disease in individuals with SCI.

Wu et al. found in an animal study that one day of methylprednisolone, at a dose comparable to those routinely employed in clinical practice immediately after SCI, resulted in marked atrophy of functionally intact muscle above the level of lesion, and worsened atrophy of paralyzed muscle below the level of lesion; this was associated with elevations in the expression of four genes involved in pathways associated with muscle atrophy.

To try to standardize care and improve communication and comparison between centers, testing and data sets have been developed. Several manuscripts in this issue look critically at some of these. Anderson et al. found, in a multi-center, prospective, cohort study, the Spinal Cord Independence Measure (SCIM III) to be a reliable and valid measure of functional change in SCI. However, improved scoring instructions and a few modifications to the scoring categories may reduce variability between raters and enhance clinical utility. Aidinoff et al. found that calculated discharge SCIM III scores can be used as target values for functional achievements at various neurological levels in patients after American Spinal Injury Association Impairment Scale (AIS) grade A spinal cord lesions (SCL). They are generally, but not always, inversely correlated with SCL level. Juul et al. describe that most items within the International Spinal Cord Injury Bowel Function Data sets have acceptable inter-rater reliability and are useful tools for data collection in international clinical practice and research. However, minor adjustments are recommended. Pernot et al. found the inter-rater reliability and validity of the test-table-test, with which Paralympic sports participants involved in Nordic sit-ski sports may be classified, very acceptable. But again some refinements are warranted.

Schoenfeld et al. characterized the incidence of SCI within the population of the United States military from 2000-2009 and defined potential risk factors for the development of SCI.

Several manuscripts deal with outcome: Scivoletto et al. compared the neurological and functional outcomes of patients with ischemic spinal cord injury (ISCI) and traumatic spinal cord injury (TSCI) in a retrospective study and found a diagnosis of ischemia and trauma is not a determinant of neurological and functional recovery in SCI patients. The outcome of the patients is influenced by age, lesion level, and AIS level. New et al. compared the demographic characteristics and rehabilitation outcomes for both non-traumatic and traumatic SCI patients admitted into either specialized or non-specialized rehabilitation units. There are differences in the characteristics of both groups. Non-traumatic patients admitted to a spinal centre have greater functional gain. Jaspers Focks-Feenstra et al. found that in the Netherlands the majority of persons with tetraplegia who underwent reconstructive upper extremity surgery were satisfied with the results, related to improvement in activities and occupation. Findings from Lauer et al. suggest that cycling with and without electrical stimulation may be beneficial for skeletal health in pediatric SCI. As a further result of their work on reemployment in Malaysia, Ramakrishnan et al. state that a rehabilitation team need to consider return to employment as a realistic goal even many years after SCI. Gajraj-Singh shows that being a primary caregiver of a SCI person contributes to caregiver burden and psychological distress, which should also be considered in the provision of care.

Two interesting case reports are also included.

Enjoy reading. 